\title{
Calcitonin gene-related peptide: the new era of migraine therapy
}

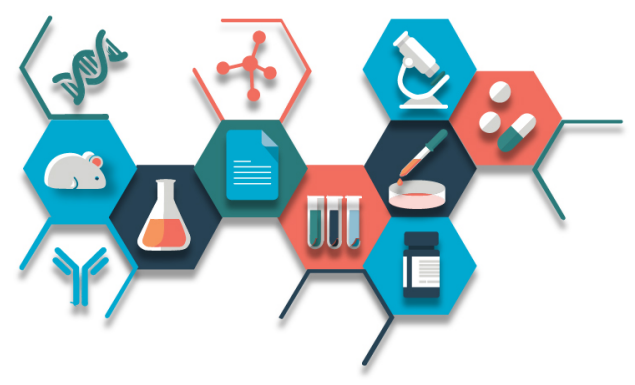

\author{
Amanda Macone ${ }^{1}$ \& Stewart J Tepper*,1 \\ ${ }^{1}$ Headache Center, Department of Neurology, Dartmouth-Hitchcock Medical Center Lebanon, NH 03766, USA \\ *Author for correspondence: sjtepper@gmail.com
}

\begin{abstract}
"The first gepant will likely be approved by the FDA by the end of 2019. Initial use will likely be targeted toward individuals with higher cardiovascular risk, as they do not cause vasoconstriction, or in those who did not tolerate or respond to triptan therapy."”
\end{abstract}

First draft submitted: 16 May 2019; Accepted for publication: 20 May 2019; Published online: 11 October 2019

Calcitonin gene-related peptide (CGRP) is a 37-amino acid neuropeptide [1] that functions by binding to a seven transmembrane G-protein coupled receptor (calcitonin-like receptor), and a single transmembrane protein (receptor activity-modifying protein 1) [2]. These CGRP receptors are located in sites believed to be involved in migraine pathogenesis. Levels of CGRP rise during migraine and cluster headache $(\mathrm{CH})$ attacks and decline following treatment [3,4]. CGRP antagonists appear to be effective in prevention and acute treatment of episodic migraine (EM) and chronic migraine (CM) and may be useful in the management of other primary headache disorders. This brief review discusses evidence supporting use of CGRP antagonists in migraine management, as well as ongoing and future research.

The first CGRP antagonists were the gepants, which are small molecule CGRP receptor antagonists. Seven gepants have been studied in migraine; IV olcegepant was not further developed due to formulation issues [5] and development of BI 44370 TA, MK-3207 and telcagepant were discontinued due to hepatotoxicity. Rimegepant, ubrogepant and atogepant do not appear to cause hepatotoxicity and are in differing stages of development.

Rimegepant has one published, positive Phase II randomized controlled trial (RCT) for acute treatment of EM [6], and three positive completed Phase III RCTs that are not yet published (as of May 2019) for acute EM treatment [7,8]. Studies 301 and 302 compared rimegepant $75 \mathrm{mg}$ tablet to placebo; preliminary data appear significant with 2-h pain freedom for rimegepant in 19.2 and $19.6 \%$ of participants in studies 301 and 302 compared with 14.2\% ( $\mathrm{p}<0.03)$ and 12\% ( $<<0.001)$ for placebo. Freedom from most bothersome symptom (MBS) occurred in 36.6 and 37.6\% of participants, compared with 27.7\% (p < 0.002) and 25.2\% (p < 0.0001) for placebo [7]. Rimegepant $75 \mathrm{mg}$ oral dissolving tablet is in development and preliminary data were also significant for primary end points of pain freedom at $2 \mathrm{~h}(21.2 \%)$ and freedom from MBS at $2 \mathrm{~h} \mathrm{(35.1 \% ),} \mathrm{compared} \mathrm{with}$ $10.9 \%(p<0.0001)$ and 26.2\% ( $=0.0009)$ for placebo [8]. On 18 March 2019, Biohaven secured a priority review voucher for their new drug application for Rimegepant Zydis oral dissolving tablet, entitling them to an expedited review by the US FDA [9]. There is also a Phase II/III trial for BHV-3500, a third-generation intranasal CGRP-receptor antagonist, for acute treatment and prevention of migraine [10].

Ubrogepant has one positive published Phase II study [11] and two released but unpublished positive Phase III RCTs, ACHIEVE 1 and ACHIEVE 2, for acute treatment in EM. Pain freedom at $2 \mathrm{~h}$ for Ubrogepant 50 and $100 \mathrm{mg}$ doses were $19.2 \%(\mathrm{p}=0.0023)$ and $21.2 \%(\mathrm{p}=0.0003)$, versus $11.8 \%$ for placebo. Relief from MBS at 2 $\mathrm{h}$ was $38.6 \%(\mathrm{p}=0.0023)$ and $37.7 \%$ ( $\mathrm{p}=0.0023$ ) versus $27.8 \%$ for placebo. The $25 \mathrm{mg}$ dose in ACHIEVE 2 was not significant for freedom from MBS at $2 \mathrm{~h}$ compared with placebo [12,13]. The Ubrogepant new drug application was accepted by the FDA on 11 March 2019 [14].

Atogepant has a positive Phase II/III study assessing the efficacy and safety of various doses of atogepant (10$120 \mathrm{mg}$ total daily) for the prevention of EM [15], and two active Phase III trials assessing the safety and tolerability of atogepant prevention of EM [16] and CM [17].

Pain freedom rates at $2 \mathrm{~h}$ for the gepants appear to be similar to triptans with slower onset, such as naratriptan, with rates ranging from 19 to $21 \%$. Preliminary results do not reveal concerns for hepatic safety, with nasopharyngitis 
and upper respiratory tract infection as the most common reported adverse effects. The first gepant will likely be approved by the FDA by the end of 2019. Initial use will likely be targeted toward individuals with higher cardiovascular risk, as they do not cause vasoconstriction, or in those who did not tolerate or respond to triptan therapy.

Following gepant development, monoclonal antibodies (MABs) targeted against the CGRP receptor and ligand itself were created. There are currently three FDA approved CGRP MABs, with one still in development; erenumab is targeted against the canonical CGRP receptor and galcanezumab, fremanezumab and eptinezumab target the CGRP ligand. MABs have a longer half-life than the gepants and are cleared through the reticuloendothelial system [3].

Erenumab is the only fully human MAB and targets the $\alpha$ CGRP receptor [18]. It was the first MAB approved for prevention of EM and CM on 17 May 2018, based on one regulatory trial in CM prevention [19], and two Phase III trials in EM prevention [20,21]. Erenumab is a once monthly subcutaneous injection given in a 70 or $140 \mathrm{mg}$ dose. There is an ongoing 5 -year safety and tolerability study. Data released at 64 weeks revealed $65 \%$ of participants reported $\geq 50 \%$ reduction in mean monthly migraine days (MMDs), $42 \%$ reported $\geq 75 \%$ reduction in MMDs and 26\% reported 100\% reduction in MMDs for periods of at least 30 days [22]. Erenumab appears effective in participants who failed prior preventive agents. The Liberty Trial compared once monthly $140 \mathrm{mg}$ erenumab to placebo in EM patients who had failed $\geq 2-4$ preventive agents. Results revealed $\geq 50 \%$ reduction MMDs in $30.3 \%$, compared with $13.7 \%$ with placebo $(p=0.02)$ [23].

Fremanezumab was the FDA approved for prevention of EM and CM on 14 September 2018, with one positive Phase II trial for EM [3,24] and two positive Phase III trials for EM and CM [25,26]. It targets the CGRP ligand and is either a monthly $225 \mathrm{mg}$ subcutaneous injection or a quarterly $675 \mathrm{mg}$ subcutaneous injection. In trials, both $225 \mathrm{mg}$ monthly and $675 \mathrm{mg}$ quarterly dosing were superior to placebo. Patients with EM and CM are undergoing 1 year open-label extension studies to further assess efficacy and safety. Interim analysis at 6 months reports $\geq 50 \%$ reduction in monthly headache days in $61-65 \%$ with EM and $51-56 \%$ with CM $[27,28]$. Fremanezumab is recruiting for a Phase II, randomized, double-blind, placebo-controlled study for the prevention of persistent post-traumatic headache [29]. Fremanezumab was studied in regulatory trials for episodic (ECH) and chronic (CCH) cluster headache. Both studies did not meet their primary end points on interim analysis and further development for $\mathrm{CH}$ prevention was terminated [30-32].

Galcanezumab was the FDA approved for prevention of EM and CM on 27 September 2018, [3] and targets the CGRP ligand. Galcanezumab is administered subcutaneously with an initial loading dose of $240 \mathrm{mg}$ and subsequent monthly maintenance doses of $120 \mathrm{mg}$. There is a published positive Phase II trial on EM prevention [33], two published Phase III trials (EVOLVE 1 and 2) on EM prevention [34,35], and a published positive Phase III trial in CM prevention (REGAIN) [36]. There is one completed and two ongoing studies assessing long-term safety and efficacy of galcanezumab in migraine. The completed long-term Phase III study revealed monthly doses of 120 and $240 \mathrm{mg}$ led to $\geq 50 \%$ reduction in MMDs in more than $50 \%$ of participants, with reductions in average MMDs decreasing 5.6 and 6.5 days, respectively, from an average of 10.6 MMDs at baseline [37]. There is an ongoing study evaluating galcanezumab in the prevention of pediatric EM (ages 6-17) [38]. Galcanezumab was studied in two pivotal trials for prevention of $\mathrm{ECH}$ and $\mathrm{CCH}$, not meeting primary end point for $\mathrm{CCH}$. In $\mathrm{ECH}$, galcanezumab $300 \mathrm{mg}$ SQ once monthly was superior to placebo in an 8-week, double-blind RCT, reducing weekly attack frequency by 8.7 attacks (baseline 17.8) compared with 5.2 attacks (baseline 17.3) with placebo $(\mathrm{p}=0.036)$ [39]. There is an ongoing, open-label, 4-year study assessing the long-term safety of galcanezumab in $\mathrm{CH}$ [40].

Eptinezumab is the only MAB against the CGRP ligand that is administered intravenously (iv.) quarterly. It is undergoing development, with one completed Phase III clinical trial (PROMISE I) for EM prevention [41], one ongoing Phase III clinical trial (PROMISE II) for CM prevention, [42] and one open-label safety study (PREVAIL) [3]. Preliminary data from the PROMISE trials have been released, but not fully published. At 12 week in the EM trial (PROMISE 1), 49.8\% ( $\mathrm{p}=0.009)$ and $56.3 \%(\mathrm{p}<0.001)$ of participants reported $\geq 50 \%$ reduction versus $37.4 \%$ for placebo with 100 and $300 \mathrm{mg}$ infusions, respectively [41]. In the CM trial (PROMISE 2), $58 \%(\mathrm{p}<0.0001)$ and $61 \%(\mathrm{p}<0.0001)$ of patients reported $\geq 50 \%$ reduction in MMDs versus $39.3 \%$ with placebo [42].

Overall, anti-CGRP therapies appear well tolerated, with fewer side effects than prior migraine preventive agents (such as the anti-epileptics, etc.). The most common side effects reported among all agents are injection site reactions (subcutaneous medications), constipation and nasopharyngitis. Safety data for ubrogepant, atogepant, rimegepant and the MAB CGRP antagonists have not shown significant risk for hepatotoxicity. Long-term safety data, which 
include 12 months of data for erenumab and at least 6 months for the other MABs against CGRP, do not appear to show increased risk for vascular events in individuals with cardiovascular or cerebrovascular risk factors [22,27,28,37,43]. Regardless, providers should be vigilant in monitoring for adverse events as these medications are prescribed to migraineurs who may be outside the studied age groups or carry more active vascular risk factors than those included in the pivotal studies. Overall, the anti-CGRP treatments appear to be effective for the prevention of EM and CM. Going forward, it will be interesting to see if these agents are effective in other debilitating primary headache disorders.

Financial \& competing interests disclosure

Grants for research (no personal compensation): Alder, Allergan, Amgen, ATI, Dr. Reddy's, ElectroCore, eNeura, Neurolief, Scion Neurostim, Teva and Zosano. Consultant and/or advisory boards: Acorda, Alder, Alexsa, Allergan, Alphasights, Amgen, ATI, Axsome Therapeutics, BioDelivery Sciences International, Biohaven, Cefaly, Charleston Labs, Decision Resources, DeepBench, Dr. Reddy's, ElectroCore, Eli Lilly, eNeura, ExpertConnect, GLG, GSK, Guidepoint Global, Impel, M3 Global Research, Magellan Rx Management, Medicxi, Navigant Consulting, Neurolief, Nordic BioTech, Novartis, Pfizer, Reckner Healthcare, Relevale, Satsuma, Scion Neurostim, Slingshot Insights, Sorrento, Sudler and Hennessey, Supernus, Teva, Theranica, Thought Leader Select, Trinity Partners, XOC and Zosano. Royalties: Springer. Salary: Dartmouth-Hitchcock Medical Center, American Headache Society. The authors have no other relevant affiliations or financial involvement with any organization or entity with a financial interest in or financial conflict with the subject matter or materials discussed in the manuscript apart from those disclosed.

No writing assistance was utilized in the production of this manuscript.

\section{Open access}

This work is licensed under the Attribution-NonCommercial-NoDerivatives 4.0 Unported License. To view a copy of this license, visit http://creativecommons.org/licenses/by-nc-nd/4.0/

\section{References}

1. Amara SG, Jonas V, Rosenfeld MG, Ong ES, Evans RM. Alternative RNA processing in calcitonin gene expression generates mRNAs encoding different polypeptide products. Nature 298(5871), 240-244 (1982).

2. Edvinsson L. The trigeminovascular pathway: role of CGRP and CGRP receptors in migraine. Headache 57(Suppl.2), 47-55 (2017).

3. Tepper SJ. History and review of anti-calcitonin gene-related peptide (CGRP) therapies: from translational research to treatment. Headache58(Suppl. 3), 238-275 (2018).

4. Goadsby PJ, Edvinsson L. Human in vivo evidence for trigeminovascular activation in cluster headache. Neuropeptide changes and effects of acute attacks therapies. Brain 117(3), 427-434 (1994).

5. Olesen J, Diener HC, Husstedt IW et al. Calcitonin gene related peptide receptor antagonist BIBN 4096 BS for the acute treatment of migraine. N. Engl. J. Med. 350(11), 1104-1110 (2004).

6. Marcus R, Goadsby PJ, Dodick D, Stock D, Manos G, Fischer TZ. BMS-927711 for the acute treatment of migraine: a double-blind, randomized, placebo controlled, dose-ranging trial. Cephalalgia 34(2), 114-125 (2014).

7. Biohaven Pharmaceuticals. Biohaven announces successful achievement of both co-primary regulatory endpoints in two pivotal Phase III trials of rimegepant an oral CGRP receptor antagonist for the acute treatment of migraine (2018). www.biohavenpharma.com/investors/news-events/press-releases/03-26-2018

8. Biohaven Pharmaceuticals. Biohaven delivers positive Phase III results with rimegepant zydis orally dissolving tablet (ODT): rapid and lasting benefit for the acute treatment of migraine (2018). www.biohavenpharma.com/investors/news-events/press-releases/12-03-2018

9. Biohaven Pharmaceuticals. Biohaven secures priority review voucher to expedite regulatory review of rimegepant zydis ODT new drug application (2019). www. biohavenpharma.com/investors/news-events/press-releases/03-18-2019

10. Biohaven Pharmaceuticals. Biohaven enrolls first patient in Phase II/III trial of BHV-3500, third generation CGRP receptor antagonist, for the acute treatment of migraine (2019). www.biohavenpharma.com/investors/news-events/press-releases/04-01-2019

11. Voss T, Lipton RB, Dodick DW et al. A Phase IIb randomized, double-blind, placebo-controlled trial of ubrogepant for the acute treatment of migraine. Cephalalgia 36(9), 887-898 (2016).

12. Allergan. Allergan announces positive top line Phase III results for ubrogepant - an oral CGRP receptor antagonist for the acute treatment of migraine (2018). www.allergan.com/news/news/thomson-reuters/allergan-announces-positive-top-line-phase-3-resul

13. Allergan. Allergan announces second positive Phase III Clinical trial for ubrogepant - an oral CGRP receptor antagonist for the acute treatment of migraine (2018). www.allergan.com/News/News/Thomson-Reuters/Allergan-Announces-Second-Positive-Phase-3-Clinica

14. Allergan. Allergan announces FDA acceptance of new drug application for ubrogepant for the acute treatment of migraine (2019). www.allergan.com/news/news/thomson-reuters/allergan-announces-fda-acceptance-of-new-drug-appl 
15. Allergan. Allergan's oral CGRP receptor antagonist atogepant demonstrates robust efficacy and safety in episodic migraine prevention in a Phase IIb/III clinical trial (2018). www.allergan.com/news/news/thomson-reuters/allergan-s-oral-cgrp-receptor-antagonist-atogepant

16. To evaluate the safety and tolerability of treatment with atogepant $60 \mathrm{mg}$ daily for the prevention of migraine in participants with episodic migraine, NCT03700320 (2019). https://clinicaltrials.gov/ct2/show/NCT03700320

17. Efficacy, safety, and tolerability of atogepant for the prevention of chronic migraine, NCT03855137 (2019). https://clinicaltrials.gov/ct2/show/NCT03855137

18. Shi L, Lehto SG, Zhu DX et al. Pharmacologic characterization of AMG 334, a potent and selective human monoclonal antibody against the calcitonin gene-related peptide receptor. J. Pharmacol. Exp. Ther. 356(1), 223-231 (2016).

19. Tepper S, Ashina M, Reuter U et al. Safety and efficacy of erenumab for preventive treatment of chronic migraine: a randomized, double-blind, placebo-controlled Phase II trial. Lancet Neurol. 16(6), 425-434 (2017).

20. Goadsby PJ, Reuter U, Hallstrom Y et al. A controlled trial of erenumab for episodic migraine. N. Engl. J. Med. 377(22), 2123-2132 (2017).

21. Dodick DW, Ashina M, Brandes JL et al. ARISE: a Phase III randomized trial of erenumab for episodic migraine. Cephalalgia 38(6), 1026-1037 (2018).

22. Ashina M, Dodick D, Goadsby PJ et al. Erenumab (AMG 334) in episodic migraine: interim analysis of an ongoing open-label study. Neurology 89(12), 1237-1243 (2017).

23. Reuter U, Goadsby PJ, Latneri-Minet M et al. Efficacy and tolerability of erenumab in patients with episodic migraine in whom two-to-four previous preventative treatments were unsuccessful: a randomized, double-blind, placebo-controlled, Phase IIIb study. Lancet 392(10161), 2280-2287 (2018).

24. Bigal ME, Dodick DW, Rapoport AM et al. Safety, tolerability, and efficacy of TEV-48125 for preventive treatment of high-frequency episodic migraine: a multicentre, randomised, double-blind, placebo-controlled, Phase IIb study. Lancet Neurol. 14(11), 1081-1090 (2015)

25. Silberstein SD, Dodick DW, Bigal ME et al. Fremanezumab for the preventive treatment of chronic migraine. N. Engl. J. Med. 377(22), 2113-2122 (2017).

26. Dodick DW, Silberstein SD, Bigal ME et al. Effect of fremanezumab compared with placebo for prevention of episodic migraine: a randomized clinical trial. JAMA 319(19), 1999-2008 (2018).

27. Brandes J, Yeung PP, Cohen JM et al. Long-term impact of fremanezumab on response rates, acute headache medication use, and disability in patients with episodic migraine: interim results of a one-year study [abstract no. PS35]. Headache 2018, 58(Suppl. 2), 170 (2018).

28. McAllister P, Yeung PP, Cohen JM et al. Long-term impact of fremanezumab on response rates, acute headache medication use, and disability in patients with chronic migraine: interim results of a one-year study [abstract no. PF14]. Headache 58(Suppl. 2), 86-87 (2018).

29. A study to test if fremanezumab reduces headache in patients with posttraumatic headache (PTH), NCT03347188 (2019). https://clinicaltrials.gov/ct2/show/NCT03347188

30. A study to evaluate the efficacy and safety of TEV-48125 (Fremanezumab) for the prevention of episodic cluster headache (ECH), NCT02945046 (2019). https://clinicaltrials.gov/ct2/show/NCT02945046

31. Teva Pharmaceutical Industries Ltd. Teva provides update on clinical trial of fremanezumab for use in chronic cluster headache (2018). www.tevapharm.com/news/teva_provides_update_on_clinical_trial_of_fremanezumab_for_use_in_chronic_cluster_headache_06_18.aspx

32. Teva Pharmaceutical Industries Ltd. Teva announces update on fremanezumab clinical development for use in episodic cluster headache (2019). www.tevapharm.com/news/teva_announces_update_on_fremanezumab_clinical_development_for_use_in_episodic_cluster_heada che_04_19.aspx

33. Dodick DW, Goadsby PJ, Spierings EL, Scherer JC, Sweeney SP, Grayzel DS. Safety and efficacy of LY2951742, a monoclonal antibody to calcitonin gene-related peptide, for the prevention of migraine: a Phase II, randomised, double-blind, placebo-controlled study. Lancet Neurol. 13(9), 885-892 (2014).

34. Staufer VL, Dodick DW, Zhang Q et al. Evaluation of Galcanezumab for the prevention of episodic migraine: the EVOLVE-1 randomized clinical trial. JAMA Neurol. 75(9), 1080-1088 (2018).

35. Skljarevski V, Matharu M, Millen BA et al. Efficacy and safety of galcanezumab for the prevention of episodic migraine: results of the EVOLVE-2 Phase III randomized controlled clinical trial. Cephalalgia 38(8), 1442-1454 (2018)

36. Detke HC, Goadsby PJ, Wang S, Friedman DI, Selzler KJ, Aurora SK. Galcanezumab in chronic migraine: The randomized, double-blind, placebo-controlled REGAIN study. Neurology 91(24), 2211-2221 (2018).

37. Camporeale A, Kudrow D, Sides R et al. A Phase III, long-term, open-label safety study of Galcanezumab in patients with migraine. BMC Neurol. 18(1), 188 (2018).

38. A study of galcanezumab (LY2951742) in participants 6 to 17 years of age with episodic migraine (REBUILD), NCT03432286 (2019). https://clinicaltrials.gov/ct2/show/NCT03432286 
39. Martinez JM, Goadsby PJ, Dodick D et al. Study CGAL: a placebo-controlled study of galcanezumab in patients with episodic cluster headache: results from the 8-week double-blind treatment phase. Presented at: 12th European Headache Federation Congress. Florence, Italy. 28-30 September 2018

40. A study of LY2951742 (galcanezumab) in participants with cluster headache, NCT02797951 (2019). https://clinicaltrials.gov/ct2/show/NCT02797951

41. Saper JR, Lipton RB, Kudrow DB et al. A Phase III, Randomized, Double-blind, Placebo-Controlled study to evaluate the effectiveness and safety of eptinezumab in frequent episodic migraine prevention: primary results of the PROMISE 1 (PRevention Of Migraine via Intravenous eptinezumab Safety and Efficacy 1) Trial. PO-01-194. Cephalalgia 37(Suppl. 1), 337 (2017).

42. Lipton RB, Saper J, Ashina M et al. A Phase III, Randomized, Double-blind, Placebo-Controlled study to evaluate the efficacy and safety of eptinezumab for the preventive treatment of chronic migraine: results of the PROMISE-2 (PRevention Of Migraine via Intravenous eptinezumab Safety and Efficacy-2) Trial. Presented at: American Academy of Neurology. CA, USA, 21-27 April 2018.

43. Ning X, Faulhaber N, Lang $\mathrm{N}$ et al. Fremanezumab cardiovascular safety profile: pooled data from placebo-controlled and long-term studies (abstract no. PS41). Headache 58(Suppl. 2), 174-175 (2018). 